How Apps-Embedded Assessment can contribute to learning outcomes mapping

\title{
Eduardo Galembeck
}

Departamento de Bioquímica, Instituto de Biologia, Unicamp, São Paulo, Brazil

Apps can be designed to provide usage data, and most of them do. These usage data are usually used to map users interests and to deliver more effective ads that are more likely to result in clicks, and sales. We have applied some of these metrics to understand how it can be used to map students' behavior using educational software. We tested both Google Analytics, and a system we have developed to map learning outcomes and students engagement. Embedded assessment were implemented in app used to teach: 1) Metabolic Pathways; 2) Protein Synthesis, 3) Cell Structure, and 4) Concepts from techniques used in a Biochemistry Lab course. Our preliminary results show that this approach provides valuable information about class outcomes that can be used for both summative and formative assessments. 